\title{
Niccolò Ugo Foscolo in Grecia: prolegomena
}

Niccolò Ugo Foscolo en Grèce : prolégomènes

Niccolò Ugo Foscolo in Greece: Prolegomena

\section{Francesca Sensini}

\section{(2) OpenEdition}

\section{Journals}

\section{Edizione digitale}

URL: http://journals.openedition.org/cei/2507

DOI: 10.4000/cei.2507

ISSN: 2260-779X

\section{Editore}

UGA Éditions/Université Grenoble Alpes

\section{Edizione cartacea}

Data di pubblicazione: 30 juin 2015

Paginazione: 201-215

ISBN: 978-2-84310-289-9

ISSN: 1770-9571

Notizia bibliografica digitale

Francesca Sensini, «Niccolò Ugo Foscolo in Grecia: prolegomena», Cahiers d'études italiennes [Online], 20 | 2015, online dal 01 janvier 2017, consultato il 26 mars 2021. URL: http://journals.openedition.org/ cei/2507 ; DOl: https://doi.org/10.4000/cei.2507 


\title{
NICCOLÒ UGO FOSCOLO IN GRECIA: PROLEGOMENA
}

\author{
Francesca Sensini \\ Université Nice Sophia Antipolis
}

Pianse tua patria, o splendissim'alma il dì che il tuo partir da lei le spiacque; e pianse poscia, e invidiò la palma, che italica, e non sua, tanto ti piacque. ${ }^{\mathrm{I}}$

\section{La patria di Foscolo: destino e scelta}

Affrontare, se pur preliminarmente, il tema della ricezione di Foscolo in Grecia implica di necessità alcune riflessioni a partire da dati biografici, e anche anagrafici, per chiarire i rapporti, anche materiali, tra il Foscolo e la sua terra natale. Se ci basassimo unicamente su questi ultimi, dovremmo concordare con Spiridon De Viasis (I843-1927), studioso di questioni genealogiche foscoliane, che deduceva inequivocabilmente da essi la seguente conclusione: «il padre del Foscolo era corcirese; l'avo corcirese; il bisavo di Candia: tutti Greci: anche il Foscolo, quindi, è greco».

Se invece rivolgiamo la nostra attenzione ai dati linguistico-letterari — alla scelta dell' "italica palma» per riprendere l'espressione di Solomòs, conterraneo del Foscolo - è chiaro come l'autore appartenga a pieno diritto alla letteratura italiana ma non altrettanto alla greca, non avendo egli mai scritto nulla, al di là di qualche stralcio di corrispondenza personale, nella sua lingua madre.

Nella dedica Alla città di Reggio che precede la prima edizione dell'ode a Bonaparte Liberatore (I797), Foscolo stesso ci fornisce gli elementi

I. D. Solomòs, In morte di Ugo Foscolo, vv. I-4. 
essenziali per riconsiderare tutti questi dati e, con essi, la sua identità d'uomo e di scrittore:

Giovane, qual mi son io, nato in Grecia, educato fra Dalmati, e balbettante da soli quattr'anni in Italia, nè dovea, nè poteva cantare ad uomini liberi ed Italiani. Ma l'alto genio di Libertà che m'infiamma, e che mi rende Uomo, Libero, e Cittadino di patria non in sorte toccata ma eletta, mi dà i diritti dell'Italiano ${ }^{2}$.

Questa dichiarazione mette in luce tutta la complessità di un'identità mista e rivendicata come tale — identità nazionale, linguistico-culturale ed estetica insieme - proiettandola su due piani complementari: da un lato la «sorte», ineluttabile, col suo portato di gloria e di tragedia, dall'altro la volontà di una scelta — la "patria eletta» — vissuta con la passione della coerenza.

La sorte volle dunque che Foscolo nascesse in Grecia, nella ionica Zante, da madre greca, di religione e cultura ortodossa, Diamantina Spathì, e da padre di lontana origine veneziana, Andrea Foscolo. È importante ricordare che la famiglia Foscolo si era insediata ormai da più di un secolo in Grecia - più precisamente a Creta - per poi trovare rifugio a Corfù, nello Ionio, nel I669, in seguito alla caduta dell'isola in mano ottomana ${ }^{3}$.

Come per tutte le famiglie sufficientemente abbienti per far studiare i propri figli, i legami dei Foscolo con la Repubblica di Venezia riguardarono essenzialmente la formazione universitaria. Come il nonno, Niccolò, il padre dell'autore studiò e si laureò in medicina a Padova per poi rientrare nell'Eptaneso e prendere servizio a Zante. Qui conobbe la moglie, che sposò "conforme il rito della Santa Madre Chiesa apostolica romana» ${ }^{4}$ nel 1777 e lì nacquero, battezzati cattolici, nell'ordine, Niccolò, Rubina e Gian Dionisio; solo il più piccolo, Costantino Angelo, vide la luce a Spalato, in Dalmazia.

Nell'ambiente in cui il nostro autore visse fino ai suoi quindici anni si parlava greco - e la sua variante dialettale isolana - e dialetto veneziano; la lingua italiana era presente come codice scritto di una cultura raffinata cui la madre stessa di Foscolo aveva potuto accedere. Secondo De Viasis, infatti, nonostante le scarse sostanze della famiglia Spathìs, il padre di

2. EN II, p. 33I.

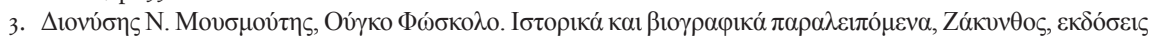
Трі́цорфо, 20Iо, р. I5.

4. Così recita il documento, in lingua italiana, conservato nell'archivio di Zante e datato 24 aprile 1777 (ivi, pp. 22-23). 
Diamantina tenne a che gli otto figli, sei femmine e due maschi, imparassero «la lettura del greco e dell'italiano, l'aritmetica e la scrittura»'.

A fronte dell'educazione in lingua greca - lingua che Foscolo continuò a usare per tutta la vita ${ }^{6}$ - vi era dunque una cultura e una lingua d'elezione, che egli non padroneggiava affatto quando si trasferì a Venezia - $\tau \rho \alpha v \lambda i \zeta \omega$, «balbetto», è il verbo che ricorre, in riferimento all'uso dell' $i$ taliano, in una lettera a Melchiorre Cesarotti del I4 maggio I795 - e che studiò con volontà e amore instancabili ${ }^{7}$. Si trattava, in realtà, di una vera e propria lingua straniera; per di più, in quanto codice poetico, altamente artificiale e indipendente tanto dalla lingua del parlare quotidiano quanto dalla lingua della prosa.

Alla luce di queste considerazioni è chiaro come sia indispensabile tenere conto della "particolare condizione interculturale» ${ }^{8}$ del Foscolo per poterne illuminare la figura e l'opera. L'identità anagrafica non è in realtà che un fatto esteriore: determinare l'appartenenza univoca a una nazione o a un'altra, a una terra o a un altra (italiano o greco, veneziano o zantiota, corcirese o cretese) può rispondere a questioni estrinseche di gloria nazionale o nazionalistica - e questo aspetto pesò non poco sul Foscolo nell'appropriazione che di lui fece il nostro Risorgimento - ma non soddisfa in alcun modo l'esigenza di definire il complesso ordito linguistico-culturale su cui l'autore ha tessuto la propria trama. Non a caso gli stessi studiosi greci del Foscolo tengono a sottolineare il fatto che resti ancora «da affrontare l’analisi antropologica della sua cultura primigenia» ${ }^{9}$.

5. Ivi, p. I9. In contrasto con questa testimonianza, Maria Antonietta Terzoli sostiene che la madre del Foscolo non leggesse i caratteri latini ma non fornisce ulteriori precisazioni al riguardo (M. A. Terzoli, Scrittori italiani nati fuori d'Italia: il caso di Foscolo e di Ungaretti, in Id., Con l'incantesimo della parola. Foscolo scrittore e critico, Roma, Edizioni di storia e letteratura, 2007, p. 228). Salvo altra indicazione, le traduzioni dal greco sono mie. Tengo a ringraziare sinceramente la collega Amalia Kolonia che, pur itinerante in Grecia, ha preso tempo per correggermi, consigliarmi e incoraggiarmi nel mio percorso. Altrettanta gratitudine va a Irini Leonti, insegnante di greco presso la Comunità greca ortodossa di Nizza Saint Spyridon, per la consulenza linguistica e la preziosa collaborazione in qualità di mediatrice culturale.

6. Nella sua Vita di Ugo Foscolo, edita a Lugano nel I830, Giovanni Pecchio afferma che Foscolo parlasse il greco "con facilità». Filippo Maria Pontani, tuttavia, nel suo esame degli scritti in greco del Foscolo, avanza riserve sulla solidità di questa conoscenza alla luce «della negligenza pressoché assoluta delle norme ortografiche, tanto più singolare in uno studioso assiduo e profondo del greco classico» e conclude che "la verità è che il Foscolo aveva una certa capacità di esprimersi in neogreco, contratta dall'infanzia [dall'ossa...] ma ne aveva una conoscenza letteraria presso che nulla» (F. M. Pontani, Foscolo e il greco moderno, Roma, Italo Graeca, 1964, p. 28).

7. Vedi C. Dionisotti, Venezia e il noviziato del Foscolo, Firenze, Sansoni, 1967.

8. M. A. Terzoli, Con l'incantesimo, cit., p. 22I.

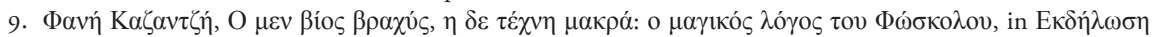

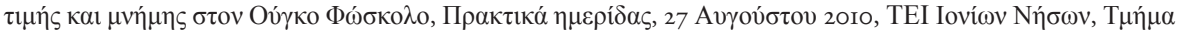

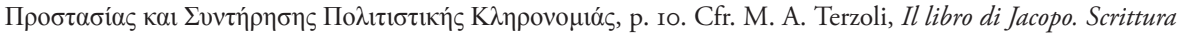
sacra nell'Ortis, Roma, Salerno, I988, pp. 5I-52. 


\section{«Sacre le reliquie $»$ : la contesa per le spoglie del poeta}

In attesa di poter approfondire una ricerca tanto complessa e appassionante, mi sono avventurata sulle tracce dell'autore - del suo nome, della sua immagine vulgata - nella sua terra natale dagli anni successivi alla morte fino ai giorni nostri. Allora, come oggi d'altronde, la prima questione associata al Foscolo in Grecia è la contesa — se così si può dire, con termine omerico - per le sue spoglie mortali ${ }^{\mathrm{I}}$.

Circa due mesi dopo la sua scomparsa, il i9 novembre 1827, nella chiesa cattolica di San Marco a Zante fu celebrata una messa in onore del Foscolo. Il poeta Dionisios Solomòs pronunciò il suo Elogio di Ugo Foscolo $^{\text {II }}$ accompagnato dal sonetto In morte di Ugo Foscolo ${ }^{12}$. In un suo recente contributo, lo studioso greco Gheràsimos Zoras sottolinea il fatto che i primi versi del sonetto costituiscano la risposta al motivo - e ossessione foscoliana - della «illacrimata sepoltura». Le lacrime della madre patria sono antiche e risalgono al primo distacco; distacco definitivo e irrimediabile, anche post mortem:

Solomòs smentisce i timori del poeta, mettendo in evidenza come gli zantioti non abbiano atteso la sua morte per piangerlo; al contrario, il loro compianto cominciò quando egli lasciò l'isola per rendersi illustre all'estero. In particolare, la sepoltura diventa ulteriore motivo di dolore per la patria, non potendo essa averlo accanto neppure da morto $^{\text {13 }}$.

Il tema foscoliano della «illacrimata sepoltura» si intreccia dunque, nei versi di Solomòs, colla vicenda della sepoltura negata, vissuta dolorosamente dai connazionali del Foscolo per il suo valore altamente simbolico e per il sentimento di ingiustizia, verso il poeta prima ancora che verso la Grecia, che essa suscitò fin dal suo inizio.

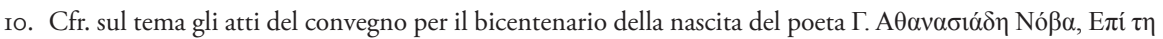

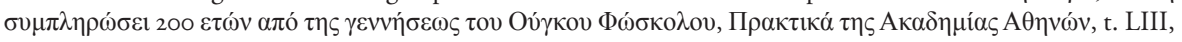
1978 .

II. D. Solomòs, Elogio di Ugo Foscolo, a cura di C. Brighenti, Torino, 1934. Alla seguente edizione del

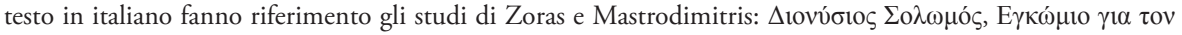

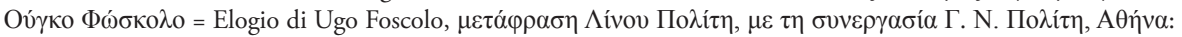

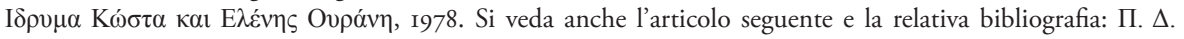

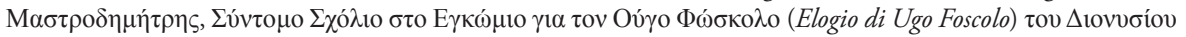

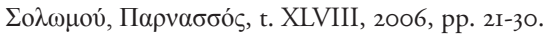

I2. Il sonetto e l'elogio funebre si trovano, unicamente in traduzione greca, negli opera omnia di D. Solomòs,

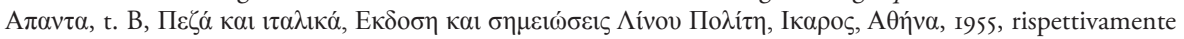

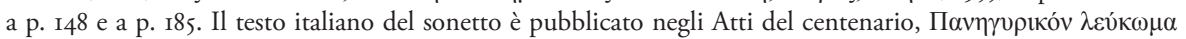

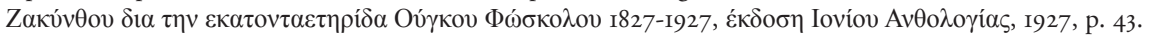

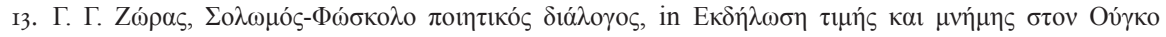

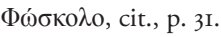


La volontà di procedere al rimpatrio della salma del Foscolo non mancò fin dai primissimi tempi successivi alla morte ma non condusse ad alcuna iniziativa concreta. Il cugino del poeta, Aloìsios Kùrtsolas si attivò rapidamente per il trasferimento della salma dal cimitero di Chiswick a Zacinto ma per ragioni ignote, pur essendo stati raccolti i fondi necessari a realizzare il trasporto, nulla ne seguì. Nel 1905, ritornando sulla questione in un articolo dedicato al Foscolo, lo storico Marinos Siguros afferma che in quell'occasione «i Greci dormirono». A controbilanciare questa constatazione Siguros sottolinea una realtà di altro segno, immutabile, che va ben oltre l'esito penoso di tutta la vicenda: «accanto ai mausolei di Dante e Michelangelo si trova anche una gloria ellenica» ${ }^{14}$.

In una lettera del I84I il deputato zantiota Ioannis Domeneghinis rispose a un giovane italiano che lo interrogava sulle iniziative in memoria del Foscolo nella sua terra natale. La replica è amara:

Sono senza parole e mi meraviglio ogni volta che richiamo alla mente il fatto che, se pure il nome di Foscolo è ammirato da tutti con parole celesti, non lo è affatto con gli atti $[\ldots]$ noi siamo degni di biasimo e giustamente gli stranieri ci criticano e denigrano quando constatano questa enorme stupidità ${ }^{15}$.

In quella che pareva una generale situazione d'inerzia delle autorità greche, nel 1870 giunse la notizia che l'Italia aveva richiesto al governo inglese il trasferimento della salma del poeta nel pantheon laico di Santa Croce. A questo annuncio non mancarono espressioni di indignazione: il Conte Spiridon Romas, residente a Napoli, protestò pubblicamente contro l'iniziativa del governo italiano, pur criticando con triste ironia la passività e i ritardi dei compatrioti ${ }^{16}$.

La protesta di Romas non fu isolata. Lo stesso anno lo storico dell'Eptaneso Panaghiotis Chiotis pubblicò sul giornale $\mathrm{H} \Delta \eta \mu$ ๘ぇкท́ una lettera al sindaco di Zacinto, Franghiskos Tzulatis, perché facesse pressione sul governo greco esigendo il trasferimento della salma sull'isola. Come già nel 1852 Chiotis aveva reagito contro coloro che "negavano la grecità dell'anima e del pensiero foscoliano» ${ }^{17}$, così in questa lettera egli associa indissolubilmente la richiesta, seppur tardiva e probabilmente destinata a restare inascoltata, delle spoglie mortali del poeta all'espressione ufficiale «davanti all'Italia, all'Inghilterra e agli altri popoli», pari a «monumento

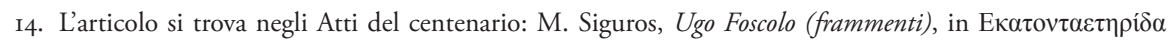

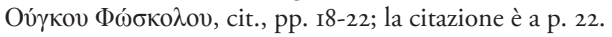

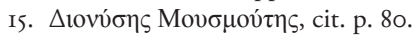

I6. Ivi, p. 82.

I7. Ivi, p. 83 . 
incrollabile», del fatto che «il geniale Foscolo è figlio di Zacinto e greco per nascita e fierezza» ${ }^{18}$.

Il sindaco di Zacinto scrisse al Primo Ministro e Ministro degli Esteri del tempo, Epaminondas Delighiorghis, chiedendo di intervenire presso le autorità inglesi e italiane; quest'ultimo trasmise la lettera a Petros Armenis Vràilas, il quale fu informato dal Ministro degli Esteri inglese che la questione era ormai definitivamente chiusa.

Quando la risposta di Armenis Vràilas fu resa pubblica, Chiotis pub-

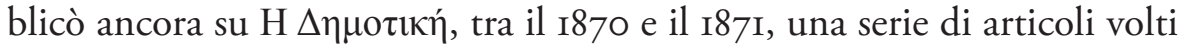
a sottolineare i diritti del Foscolo contro le pretese del governo italiano, la grecità indiscutibile del poeta e i suoi sentimenti nei confronti della madre patria. Su iniziativa dello storico, si tentarono altre mediazioni, attraverso il deputato italiano di origine greca Giorgio Tamazto-Grassetti e il console italiano a Zacinto, Costantino Messalas, fino all'iniziativa del deputato greco zantiota, Kostantinos Lombardos, che si rivolse direttamente al re Vittorio Emanuele, a Garibaldi e altre personalità italiane di sua conoscenza cercando di far valere i diritti di Zacinto.

Sempre nel I87I il poeta leucadio Anghelos Kalkanis scrisse un poema in italiano, $A$ Zacinto, dedicato al sindaco e ai consiglieri municipali di Zacinto "compatrioti del grande poeta dei Sepolcri». L'intento di Kalkanis era di esprimere solidarietà rispetto alle iniziative delle autorità dell'isola e compartecipazione al dolore per questa «seconda perdita» del poeta ${ }^{19}$.

Quando, nel maggio del I871, il commissario Angelo Bargoni si recò a Londra per l'esumazione, si verificarono difficoltà impreviste, dovute all'impossibilità di rinvenire la salma del poeta nel cimitero di Chiswich ${ }^{20}$. La stampa greca reagì a questa notizia con una malcelata soddisfazione, rivelatrice dei sentimenti dell'opinione pubblica colta e del mondo intellettuale, in particolare ionico, rispetto alla vicenda. Così leggiamo sul set-

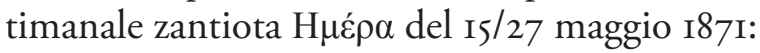

In attesa di maggiori informazioni sul chiarimento di questo mistero [scil. il mancato ritrovamento della salma], non possiamo nascondere che l'Italia, disprezzando la

\section{I8. Ivi, p. 84 .}

19. Ivi, pp. 93-95.

20. Ce ne dà notizia P. Artusi, Vita di Ugo Foscolo, Tipografia di G. Barbera, Firenze, I878, p. 210: «La sera de’ 24 maggio il comm. Peruzzi riceveva dal prefato commissario un telegramma con cui si avvertiva di sospendere i preparativi perché gli avanzi del cantor de' Sepolcri erano scomparsi, né era più possibile rintracciarli. Fatte però più diligenti ricerche nel cimitero di Chiswick, furono finalmente trovati e l'onorevole Bargoni esultante potè darne il grato annunzio al Ministro dell'Istruzione pubblica, onorevole Correnti, col seguente dispaccio: Londra, 8 giugno. Ugo Foscolo è restituito all'Italia. Fu compiuto il dissotterramento; il cadavere fu trovato in istato perfetto di conservazione dentro a due casse ben condizionate, l'autenticità ne è completamente accertata; il ministro Cadorna e buon numero d'Italiani era presente». 
precisa richiesta del poeta greco per nascita, si è stimata in diritto di non consentire al trasporto delle sue ossa nella sua isola natale, Zacinto ${ }^{21}$.

Significativo in questo senso è il fatto che l'ambasciatore greco in Italia, Andreas Kunduriotis, non abbia preso parte direttamente alle sontuose manifestazioni organizzate a Firenze per il ritorno delle spoglie del poeta, delegando il proprio segretario. Questo fatto non mancò di suscitare critiche presso la comunità greca residente in Toscana, che avrebbe desiderato una più grande rappresentanza delle autorità elleniche.

L'amarezza della Grecia rispetto a questo evento non scemerà. La questione della frustrata gloria nazionale s'intreccerà sempre, indissolubilmente, al tema del mancato rispetto delle ultime volontà del poeta. Quando, il 4 settembre I9I5 il poeta greco Kostìs Palamàs pubblicò sul

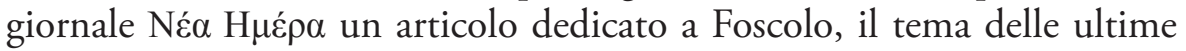
volontà del poeta "più greco per spirito di quanto fosse italiano per lingua» ricorre in toni accorati:

Foscolo nacque a Zacinto da madre zantiota e in quest' isola fiorente trascorse i primi anni dell'infanzia e dell'adolescenza. Il poeta italofono, ma più greco per spirito di quanto fosse italiano per lingua, è figlio di Zacinto, come Solomòs. Ma Solomòs rimase più fedele alla grande madre mentre il suo maestro e conterraneo di vent'anni più vecchio, espatriato prematuramente, non riuscì a rivedere la patria, e fino all'ora della sua morte lo accarezzarono sogni appassionati, e mai realizzati, di un ritorno ad essa ${ }^{22}$.

Nel 1927, in occasione del centenario della nascita del poeta, tra le altre iniziative culturali promosse in Grecia, venne pubblicata una traduzione commentata dei Sepolcri, opera di Gheòrghios Kalosghuros. La traduzione è arricchita non solo da un'introduzione al testo e da un apparato di schòlia ma anche dalla traduzione dei Cimiteri di Ippolito Pindemonte, da una nota biografica di Marinos Siguros e da un articolo di Spiros Minotos dal significativo titolo "L'anima greca di Ugo Foscolo»" ${ }^{23}$ In questo articolo lo studioso intende illustrare la «coscienza greca» del poeta riferendosi essenzialmente «alle sue stesse dichiarazioni sulle sue origini e sui suoi sentimenti per Zacinto e la Grecia» ${ }^{24}$. Minotos passa dunque in rassegna e cita, in traduzione greca, tutti i luoghi più significativi dell'epistolario di Foscolo in cui vengono evocate le sue origini, il suo amore per la patria, il

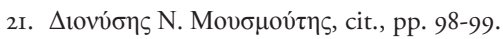

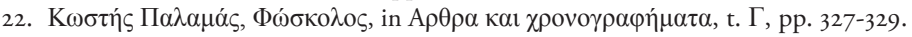

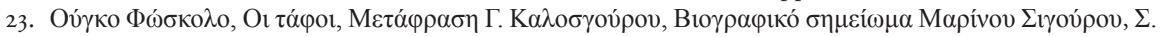

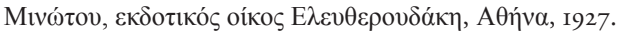

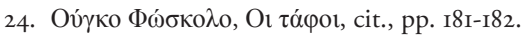




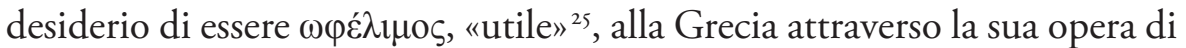
intellettuale e, naturalmente, il sogno mai abbandonato di poter tornare a vivere, e morire, a Zacinto.

\section{Il centenario della morte del poeta}

Nel 1927, dunque, ricorse il centenario della morte del poeta. In questa occasione, Grecia e Italia si accordarono per organizzare congiuntamente le celebrazioni. L'occasione del centenario fece da sfondo al viaggio a Roma dei Ministri degli Esteri e dell'Economia, Andreas Michalakòpulos e Gheòrghios Kafandaris, inviati coll'obiettivo di ottenere dei finanziamenti a beneficio della neonata democrazia ma anche per comunicare, seppur diplomaticamente, alcune rimostranze del governo greco sulla condotta delle autorità italiane nel Dodecaneso. Nel complesso la visita a Roma della delegazione greca fu fruttuosa. Nel frattempo, in Grecia, si costituiva il comitato organizzativo per i festeggiamenti foscoliani, presieduto dal Ministro degli Interni, Panaghìs Tsaldaris, e composto da personalità provenienti dal mondo accademico e dalla comunità zantiota. I due paesi si accordarono per realizzare delle celebrazioni comuni che avrebbero avuto luogo nel novembre 1927 con rappresentanti governativi,

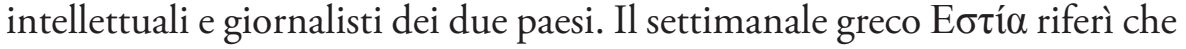
le feste si erano svolte «in un'atmosfera di alta e nobile esaltazione spirituale, creata dalla comunicazione con lo spirito di un grande come Ugo Foscolo, il quale ebbe per patria due popoli tra cui esisteva una grande affinità spirituale e intellettuale» ${ }^{26}$.

Più in generale, la stampa commentò con entusiasmo l'avvicinamento tra i due paesi, enfatizzato pubblicamente dalle autorità italiane in Grecia; il sottosegretario di Stato al Ministero della pubblica istruzione italiano, Emilio Bodrero, ebbe a sottolineare come mai nella storia europea Italia e Grecia si fossero trovate in un momento più propizio per intendersi. Nella quantità delle pubblicazioni, critiche e di corrispondenza, si distinse tut-

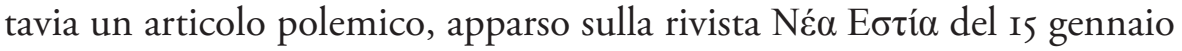
I928, in cui la giornalista Alkis Thrilos non solo mise in dubbio la sincerità

25. L'aggettivo greco è impiegato dal Foscolo in una lettera in greco arcaicizzante del $2 \mathrm{I}$ aprile $\mathrm{I} 824$, indirizzata a una personalità politica greca, I. Orlandos (Ep. III, pp. I52 sgg.). Cfr anche F. M. Pontani, Foscolo e il greco moderno, cit., p. 32.

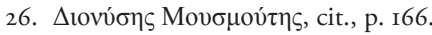


del filellenismo ${ }^{27}$ foscoliano ma sottolineò altresì il carattere «interessato» delle celebrazioni per il centenario evocando una deliberata volontà di propaganda dell'Italia fascista in territorio ellenico:

I festeggiamenti in onore di Foscolo hanno servito, in fin dei conti, interessi politici. Gli italiani volevano fare una manifestazione dell'Italia fascista nell'Eptaneso. Foscolo era solo un pretesto. Questa manifestazione gli italiani avevano deciso di farla e da soli. La Grecia prese l'iniziativa e si incaricò dell'organizzazione delle celebrazioni. Questo è l'intero segreto delle celebrazioni foscoliane ${ }^{28}$.

Su questa scia polemica un interessante e poco noto dibattito, riportato alla luce dalla recente monografia di Dionisis Musmutis dedicata a Foscolo, si accese intorno all'autore nell'ambiente della critica letteraria marxista più o meno strettamente legata al Partito comunista di Grecia, il KKE

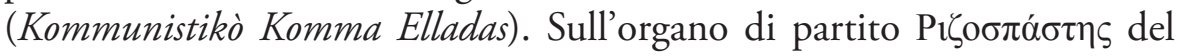
27 novembre 1927 venne pubblicato un articolo, eminentemente biografico, dedicato a Foscolo, nel quale si mettevano in evidenza l'impegno del poeta nelle lotte sociali del suo tempo e la coerenza delle sue posizioni ideologiche. L'autore, che si firmò colle iniziali N. K., era verosimilmente l'avvocato, giornalista e scrittore Nikos Katiforis.

L'articolo suscitò la reazione immediata dell'intellettuale e scrittore Petros Pikròs (pseudonimo letterario di Petros Ghunaròpulos) che scrisse una lettera aperta al giornale, in cui accusava personalmente Katiforis di voler ignorare gli scopi politici del fascismo insiti nelle celebrazioni foscoliane e di essere vicino agli «ammiratori borghesi» del Foscolo. Per quanto riguarda il poeta, Pikròs sottolinea come egli non abbia alcuna rilevanza nella letteratura neogreca, non avendo scritto che in lingua italiana, «a meno che non si debbano considerare monumenti estetici delle lettere e dei riferimenti galanti e adulatori — le sole 'opere' del poeta scritte in greco» ${ }^{29}$.

Dal punto di vista ideologico, il giudizio non è meno secco e radicalmente negativo: Foscolo ha ignorato i fermenti sociali del suo tempo e la sua fama di precursore delle rivoluzioni nazionali e di $\varphi \imath \lambda \varepsilon \lambda \varepsilon v ́ \theta \varepsilon \rho \varsigma_{\varsigma}$ è ingiustificata ${ }^{3 \circ}$; egli era piuttosto un avventuriero, $\pi \rho \alpha ́ \kappa \tau \omega \rho$, «agente», di

27. Sul tema del filellenismo italiano nel XIX secolo si vedano Garibaldi e il filellenismo italiano nel XIX secolo, Atene, Istituto italiano di cultura in Atene, 1985; Risorgimento greco e fllellenismo italiano. Lotte, cultura, arte, Mostra promossa dall'Ambasciata di Grecia e dall'Associazione per lo sviluppo delle relazioni fra Italia e Grecia, Roma, palazzo Venezia, 25 marzo-25 aprile 1986, catalogo a cura di C. Spetsieri Beschi ed E. Lucarelli, Roma, Edizioni del Sole, 1986; F. Bellucci, La Grecia plurale del Risorgimento (I82I-I9IS), Pisa, ETS, 2012.

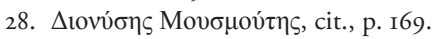

29. Ivi, p. 176 .

30. Sulla visione del Foscolo rispetto alla situazione politica in Grecia si veda, oltre agli Scritti sulle isole ionie pubblicati in U. Foscolo, EN, XIII/I, pp. I-582, la monografia dedicata alla rivoluzione greca di $\Sigma \pi v \rho i ́ \delta \omega v \Delta \varepsilon$

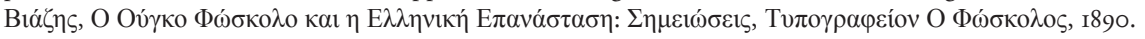


Napoleone, del re Ludovico, del governo britannico e dello Zar. Senza citare documenti o fonti, cui afferma di volersi riferire in una nota successiva, Pikròs continua su questo tono:

Non basta il documento d'identità di cittadino austriaco - di cui non so perché non ha fatto menzione il compagno N. K. - a servire come buona certificazione delle battaglie del Foscolo. Le sue negoziazioni mercantili con Koletis per tornare in Grecia, la corrispondenza col ministro dello Zar, il servilismo nei confronti del governo inglese per persuaderlo che non era un agente della Russia, la paura di condannare le turpitudini, di cui nessuno parlava, dell'Inghilterra nell'Eptaneso ${ }^{31}$, il fatto che non solo non abbia preso parte alla lotta della borghesia greca ma che abbia ignorato i moti delle plebi zantiote [...], la sua mania di evidenziare che era aristocratico e la sua falsa attestazione di discendenza dalla famiglia di Leonardo Foscolo, tutto questo testimonia degli scopi che si intrecciavano nei suoi vari movimenti ${ }^{32}$.

Nella sua risposta, del 29 novembre 1927, intitolata "Le feste foscoliane», Katiforis non difende solo se stesso dalla accuse di 'tradimento' ideologico del compagno Pikròs ma anche e soprattutto Foscolo, la coerenza della sua etica, il suo amore per la libertà e la sua dignità letteraria, concludendo sostanzialmente che l'accusatore, senza veri argomenti a sostegno della sua tesi, si limitava a diffondere calunnie:

Foscolo non fu affatto un mediocre ma appartenne ai migliori scrittori del suo tempo e i suoi servigi si sarebbero venduti caramente se si fosse mai trattato di renderli a qualcuno. Ma questo accadde? No, tutt'altro. La polizia austriaca lo sorveglia e lo ricerca. In Inghilterra si crea intorno a lui un'atmosfera ostile. Serviva talmente gli interessi britannici che gli negarono il passaporto per Zacinto affinché i suoi concittadini non prendessero coraggio dalla sua venuta e non si ribellassero a Lord Maitland, che governava despoticamente la sua patria. Servì talmente gli interessi della Russia che quando Kapodistrias, ministro dello Zar, dopo una visita in cui lo trovò in una situazione d'indigenza disperata, lo invitò in Russia dove vi erano i mezzi per mantenerlo, egli rifiutò per non sottomettersi al regime totalitario zarista. E per quando riguarda «i servigi» che rese agli interessi di Napoleone, occorre notare questo: Foscolo ammirò Napoleone come generale della Rivoluzione, e certamente gli aveva dedicato un'ode in merito. Quando tuttavia Napoleone svelò le sue ambizioni da dittatore, lo dichiarò suo nemico. E questo fu il risultato: la cattedra all'università di Pavia dove esercitava come professore fu soppressa ed egli rimase privo di risorse per vivere ${ }^{33}$.

Pikròs portò avanti la polemica antifoscoliana pubblicando sul mensile

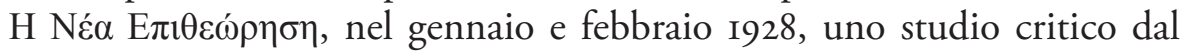

3I. Per la visione del Foscolo sulla situazione politica nell'Eptaneso e sul ruolo del governo inglese - e per

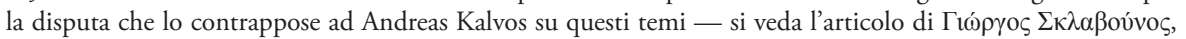

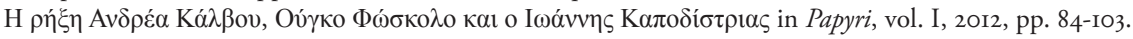

32. Ivi, pp. I73-I74.

33. Ivi, pp. I77-I78. 
significativo titolo «Foscolo senza la leggenda. Significato politico e sociale del centenario del poeta». Dionisis Musmutis fa notare che le posizioni di un intellettuale come Pikròs riflettono, in realtà, una più generale impostazione della cultura della sinistra greca tra le due guerre:

L'uso ideologico della Storia, in particolare nel periodo tra le due guerre, orienterà la Sinistra verso una forma di 'populismo', con il rifiuto, in parallelo, dei prodotti dell'arte borghese. Domina largamente il punto di vista secondo cui la borghesia ha fallito nel tentativo di rivolgersi, colla sua letteratura, alle masse popolari ${ }^{34}$.

Questa critica coinvolge non solo un autore dall'identità contesa, come Foscolo, ma anche grandi esponenti della letteratura greca di Otto e Novecento, quali Dionisios Solomòs, Andreas Kalvos, Kostìs Palamàs, Aristotelis Valaoritis, Anghelos Sikelianòs, Kostas Vàrnalis, che verrano 'riabilitati' più tardi, come nel caso emblematico di un altro zantiota, Kalvos, che divenne «un poeta-guida che, col suo esempio, indicò ai greci progressisti la via per un'azione di lotta senza compromessi e concessioni». Nel caso particolare di Foscolo, la polemica si aprì ed esaurì cogli articoli di Katiforis e Pikròs - e in particolare di quest'ultimo - senza tuttavia che essa riuscisse a contribuire in modo costruttivo alla migliore comprensione della figura e dell'opera dell'autore nella sua terra natale.

Oltre all'interesse - e alle polemiche - registrati nella stampa ellenica, il centenario della morte di Foscolo segnò un momento di ripensamento e riflessione sulla figura e sull'opera del poeta in ambito accademico e letterario. Gli Atti del centenario, prevalentemente ma non esclusivamente in lingua greca, colpiscono per la loro variegata ricchezza di contributi: dalle traduzioni della produzione lirica di Foscolo a poemi originali in onore del poeta (compresa la traduzione greca del poema Per il trasporto delle reliquie di Ugo Foscolo in Santa Croce del Carducci), dalle prose degli studiosi ai ritratti aulici e romanzeschi dei letterati, per finire con una sezione di versi e prose dedicata a Zacinto quale terra privilegiata di arte e cultura (compaiono in questa sezione anche $\mathrm{i}$ testi in inglese e in francese To Zante di Edgar Allan Poe e Zante di Paul Bourget).

Tra i contributi più prestigiosi si segnala il diffuso articolo del poeta e scrittore Kostas Varnalis sulla teoria estetica del Foscolo e, più in particolare, sulla compiuta realizzazione dell' «ideale classico» che egli riconosce nell'opera foscoliana ${ }^{35}$.

34. Ivi, pp. I83.

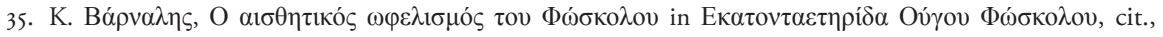
pp. 23-28. 
Sul tema della grecità del Foscolo, invece, sono particolarmente interessanti due brevi articoli di Thomàs Velianitis e Michalis Valsas. Nel primo, dal titolo Greco o Italiano, lo scrittore, dopo aver illuminato le circostanze storiche che portarono molti futuri letterati dell'Eptaneso a esprimersi in italiano, riconosce «l'anima nazionale» del Foscolo nel suo interesse e nella sua azione in favore dell' «indipendenza greca» (anch'essa ostacolata e per così dire neutralizzata dalle dolorose circostanze della sua vita):

Foscolo soffrì per la Grecia come Solomòs. Ce lo disse lo stesso Solomòs nel discorso commemorativo che pronunziò per la morte del Foscolo. Foscolo fu perso per la letteratura greca e rimase lontano dai ranghi delle lotte politiche dell'Eptaneso in ragione della sua povertà ${ }^{36}$.

Nel secondo contributo, dal significativo titolo Annessioni (in merito alla grecità del Foscolo), Valsas polemizza con toni appassionati nei riguardi di una tendenza dei suoi connazionali che sembra superare i confini del caso foscoliano per farsi generale; egli arriva ad affermare quanto segue: «temo molto che se Foscolo non avesse sangue greco nelle vene, e anche se il suo valore di poeta fosse maggiore, non lo loderemmo tanto". Al di là di questa critica generale, l'invito e l'auspicio dello scrittore sono chiari: amare l'opera dei poeti che, come Foscolo, hanno amato la Grecia pur essendosi espressi in una lingua straniera: «amiamo la loro opera: parliamone meno e leggiamola di più $[. .$.$] amiamo e ammiriamo Foscolo per$ poterlo rivendicare come nostro» ${ }^{37}$.

\section{Foscolo in Grecia oggi}

Al di là dell'interesse di singoli studiosi greci per il poeta italofono «che mai smise di sentirsi zantiota ${ }^{38}$, il centro catalizzatore della vitalità del Foscolo nella sua terra è senz'altro l'Associazione culturale Ugo Foscolo

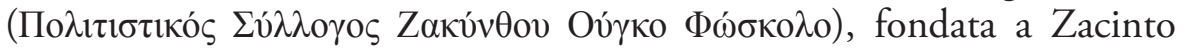
nel 2009. Lo scopo della sua nascita è «la ricostruzione della casa dell'artista italo-zantiota» ${ }^{39}$, nella forma più possibile fedele all'originale, e della biblioteca foscoliana al suo interno.

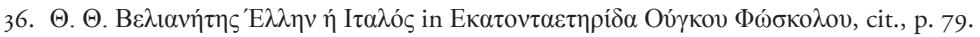

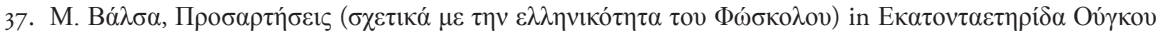

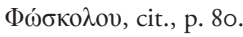

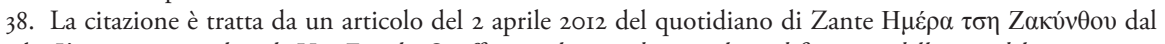
titolo L'associazione culturale Ugo Foscolo: Si affrettino le procedure per la riedificazione della casa del poeta!

39. La dichiarazione è riportata sul sito in lingua greca della rivista letteraria "Ombra dell'ombra»: <http:// www.iskiosiskiou.com/2010/o8/ugo-foscolo.html>, curata da padre Panaghiotis Kapodistrias, officiante nella cattedrale di Zante. 
Un'amara ironia, infatti, ha voluto che questo luogo fondamentale, unico segno tangibile della presenza del Foscolo sull'isola, venisse distrutto dai bombardamenti dell'aviazione italiana il 6 novembre 1940. Precedentemente la dimora, piccola e modesta, in via Odighìtrias, era parte del demanio dello stato, che la acquistò nel I886. La celebre biblio-

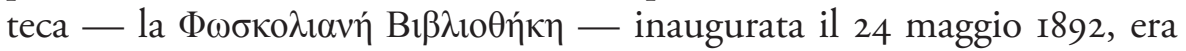
stata integrata in un secondo momento alla biblioteca comunale di Zante e andò in seguito distrutta nel terremoto del 1953. Grazie ai finanziamenti stanziati della Regione Ionia con fondi europei, l'obiettivo principe dell'associazione può essere raggiunto; si attende l'inizio dei lavori. Fra le altre ambizioni dell'associazione si annovera la costituzione, nella casa ricostruita, di un fondo bibliografico foscoliano composto da edizioni, traduzioni, monografie e articoli dedicati all'autore.

Accanto a questi progetti, dall'anno della sua nascita a oggi, l'associazione ha promosso direttamente e preso parte a eventi culturali locali in onore del Foscolo. Il 27 agosto 20Io, a Zante, si è tenuta la prima "manifestazione in onore e in ricordo" ${ }^{\circ}$ di Foscolo, con interventi di vari rappresentanti del mondo della cultura greca, come il saggista Dionisis Musmutis, i professori Fanì Kazantzì e Gheràsimos Zoras, lo scrittore Filippos Drakontaidìs e l'attrice zantiota Tzeni Rusea ${ }^{4}$.

Più di recente, il 24 febbraio 20I3, nella cattedrale di Zante, il centro culturale $A \lambda \eta \theta \omega ́ s$ ha dedicato una delle sue riunioni annuali - e più

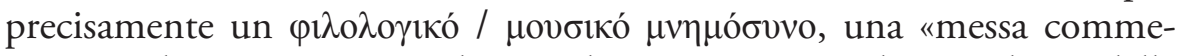
morativa letteraria e musicale» — al «poeta zantiota dei Sepolcri e delle Grazie» ${ }^{42}$. A questo evento hanno preso parte autorità ecclesiastiche - il metropolita di Dodona e di Zacinto - e politiche locali, nella persona del sindaco della città. Ai saluti dell'ambasciatore greco a Roma e del presidente del dipartimento di lingue straniere, traduzione e interpretazione dell'Università dello Ionio, sono seguiti gli interventi del presidente dell'Associazione Ugo Foscolo, Nikos Lalòtis e del presidente di A $\lambda \eta \theta \omega ́ \varsigma$, chiusi da un concerto di musica barocca ${ }^{43}$.

Sul versante delle ricerche specialistiche, l'assenza di una bibliografia dedicata all'autore ha senz'altro ostacolato lo sviluppo degli studi foscoliani in Grecia. Solo negli ultimi decenni si è registrato un interesse crescente verso l'autore con la pubblicazione di opere molto importanti, quali

40. Ibid.

4I. Cfr. supra n. 9 per gli Atti della giornata.

42. Si veda la seguente pagina del sito in lingua greca $<$ http://www.amen.gr/articleı26o9>.

43. Gli atti di questo evento sono stati pubblicati in un numero della rivista del centro culturale stesso:

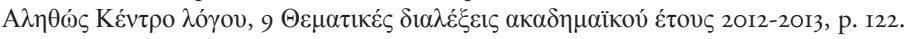


il repertorio di articoli tratti dai quotidiani e periodici dedicati a Foscolo e Kalvos di Dionisis Serras, la più recente monografia sulle traduzioni greche delle opere foscoliane di Fanì Kazantzì e naturalmente la preziosissima introduzione storica e biografica all'autore di Dionisis Musmutis, fornita di una ricca bibliografia greca e internazionale ${ }^{44}$.

\section{Conclusione}

Per concludere, questo primo tratto di cammino sulle tracce della presenza foscoliana in Grecia ci rivela un quadro animato da passioni nazionali, traversie storiche legate al processo di unificazione e stabilizzazione della democrazia nel paese e da un senso di rimpianto che dal poeta stesso, profeta di una sua sepoltura lontana - se non in terra straniera quanto meno lontano dal "petto della madre mesta» — si trasmette ai suoi compatrioti, del suo tempo come di oggi.

Fermo il fatto che Foscolo riunisce in sé «il sacro palpito della poesia greca e la fiamma dello spirito latino che partì da Zacinto per l'Italia dove volano le Muse profughe» ${ }^{45}$ - e non sfugga il senso di una fatalità storica sommamente ingiusta - è particolarmente degno di nota che la questione della lingua venga trattata come un elemento secondario, accidentale, del legame tra il poeta e la sua terra. I suoi testi vengono proposti, citati e letti pubblicamente nelle innumerevoli traduzioni che, a partire dall'Ottocento fino ad oggi, sono state realizzate dai suoi compatrioti greci italofoni ${ }^{46}$. La versione originale italiana si affianca a essi senza sollevare aspetti problematici, come un fatto legato più a circostanze

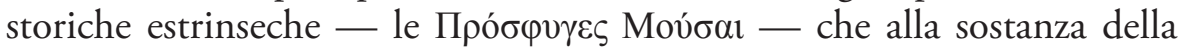
personalità e dell'arte foscoliane. Il pensiero di Nikos Veis, bizantinista dell'Università di Atene negli anni Trenta, esprime efficacemente questo

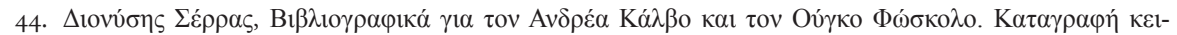

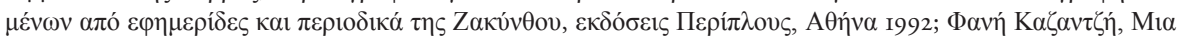

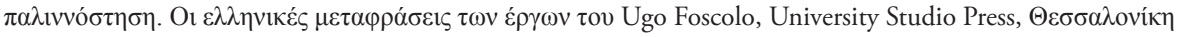

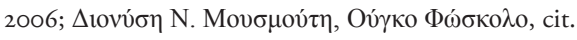

45. Sono le parole stesse di M. Minotu, studiosa zantiota del Foscolo, responsabile della casa editrice

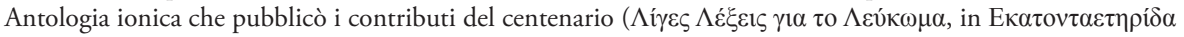

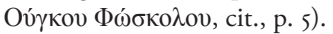

46. Per quando riguarda le traduzioni in neogreco delle opere del Foscolo, oltre a quelle presenti negli atti del centenario e riguardanti essenzialmente la produzione lirica del poeta, il romanzo Ultime lettere di Jacopo Ortis conobbe due traduzioni, la prima nel I838, di I. G. Calamogdartis, e la seconda, nel ı886, di K. Christòpulos. La traduzione di A. Politis, lodata dal Foscolo stesso, ancora in vita, non fu mai pubblicata. Innumerevoli, in

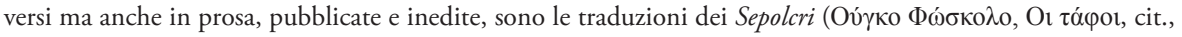
pp. 34 e 37). 
sentimento presente e costante, in filigrana, dietro ogni evocazione del Foscolo: «se la sua voce, che per noi suonò triste e cara, si fece italiana, la sua anima, la sua grande anima rimase verginalmente greca» ${ }^{47}$.

Al contrario, emerge con evidenza, in ogni intervento, discorso commemorativo o ricerca specialistica che sia, il ricordo di un voto inesaudito che rivendica, anche dalla tomba, i suoi diritti. Non a caso l'associazione culturale intitolata al poeta precisa che il progetto di ricostruzione della casa natale del Foscolo risponde essenzialmente alla volontà di porre fine all'erranza del poeta, restituendogli, con un ritardo che l'ammirazione sincera e il culto della memoria intendono medicare, la dimora della sua infanzia zantiota ${ }^{48}$.

È a partire da queste premesse culturali - e diremmo anche sentimentali - e dalle ricerche specialistiche in lingua neogreca, elaborate da un punto di osservazione particolare e complementare rispetto a quello nostro, italiano, che intendiamo proseguire, nel futuro, il nostro studio sulle tracce del Foscolo - la sua immagine, la ricezione delle sue opere, la sua influenza sugli scrittori greci - nella sua originaria e indimenticata $\mu \eta \tau \rho \iota n ́ ~ \gamma \eta$, «materna terra».

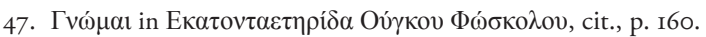

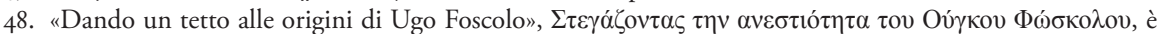
il titolo dell'articolo dedicato alle feste foscoliane del 27 agosto $2010<$ http://www.iskiosiskiou.com/20Io/o8/ ugo-foscolo.html>. 